\title{
Article \\ Expression Analysis of MaTGA8 Transcription Factor in Banana and Its Defence Functional Analysis by Overexpression in Arabidopsis
}

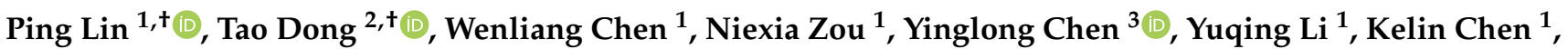 \\ Mingyuan Wang ${ }^{1, *}$ and Jianfu Liu ${ }^{1, *}$ \\ 1 Institute of Horticulture Science and Engineering, Huaqiao University, Xiamen 361021, China; \\ L_ping0509@stu.hqu.edu.cn (P.L.); wenliangchen@stu.hqu.edu.cn (W.C.); 19014087041@stu.hqu.edu.cn (N.Z.); \\ 18013087012@stu.hqu.edu.cn (Y.L.); kelin@stu.hqu.edu.cn (K.C.) \\ 2 Key Laboratory of South Subtropical Fruit Biology and Genetic Resource Utilization, Ministry of Agriculture, \\ Key Laboratory of Tropical and Subtropical Fruit Tree Research of Guangdong Province, Institution of Fruit \\ Tree Research, Guangdong Academy of Agricultural Sciences, Guangzhou 510640, China; taod2004@163.com \\ 3 The UWA Institute of Agriculture, and School of Agriculture and Environment, The University of Western \\ Australia, Perth 6009, WA, Australia; yinglong.chen@uwa.edu.au \\ * Correspondence: mywang@hqu.edu.cn (M.W.); jianfu@hqu.edu.cn (J.L.); Tel.: +86-592-6162300 (M.W. \& J.L.) \\ $\dagger$ The first two authors contributed equally.
}

check for

updates

Citation: Lin, P.; Dong, T.; Chen, W.; Zou, N.; Chen, Y.; Li, Y.; Chen, K.; Wang, M.; Liu, J. Expression Analysis of MaTGA8 Transcription Factor in Banana and Its Defence Functional Analysis by Overexpression in Arabidopsis. Int. J. Mol. Sci. 2021, 22, 9344. https://doi.org/10.3390/ ijms22179344

Academic Editor: Yong-Hwan Moon

Received: 14 July 2021

Accepted: 23 August 2021

Published: 28 August 2021

Publisher's Note: MDPI stays neutral with regard to jurisdictional claims in published maps and institutional affiliations.

Copyright: (C) 2021 by the authors. Licensee MDPI, Basel, Switzerland. This article is an open access article distributed under the terms and conditions of the Creative Commons Attribution (CC BY) license (https:// creativecommons.org/licenses/by/ $4.0 /)$.
Abstract: TGA transcription factor is a member of the D subfamily of the basic region-leucine zippers (bZIP) family. It is a type of transcription factor that was first identified in plants and is the main regulator in plant development and physiological processes, including morphogenesis and seed formation in response to abiotic and biotic stress and maintaining plant growth. The present study examined the sequence of the MaTGA8 transcription factor, the sequence of which belonged to subfamily D of the bZIP and had multiple cis-acting elements such as the G-box, TCAelement, TGACG-element, and P-box. Quantitative real time polymerase chain reaction (qRT-PCR) analyses showed that MaTGA 8 was significantly down-regulated by the soil-borne fungus Fusarium oxysporum f. sp. cubense race 4 (Foc TR4). Under the induction of salicylic acid (SA), MaTGA8 was down-regulated, while different members of the MaNPR1 family responded significantly differently. Among them, MaNPR11 and MaNPR3 showed an overall upward trend, and the expression level of MaNPR4, MaNPR8, and MaNPR13 was higher than other members. MaTGA8 is a nuclear-localized transcription factor through strong interaction with MaNPR11 or weaker interaction with MaNPR4, and it is implied that the MaPR gene can be activated. In addition, the MaTGA8 transgenic Arabidopsis has obvious disease resistance and higher chlorophyll content than the wild-type Arabidopsis with the infection of Foc TR4. These results indicate that MaTGA8 may enhance the resistance of bananas to Foc TR4 by interacting with MaNPR11 or MaNPR4. This study provides a basis for further research on the application of banana TGA transcription factors in Foc TR4 stress and disease resistance and molecular breeding programs.

Keywords: MaTGA8 transcription factor; MaNPR1; Fusarium oxysporum f. sp. cubense race 4; salicylic acid

\section{Introduction}

The TGA transcription factor is in the D subfamily of the basic leucine zipper (bZIP) family and specifically recognizes and binds to activation sequence 1 (as-1) with TGACGT as the core [1], while the leucine zipper region participates in oligomerization. The $\mathrm{N}$ terminus of the transcription factor contains the acidic activation region STDxDT that can form a dimer, and the basic region binds directly to DNA [2]. The transcription factor can activate or inhibit the transcriptional expression of downstream interaction 
genes to regulate the normal growth and development of plants under stress or nonstress. For example, ten TGA transcription factors have been isolated and identified from Arabidopsis, which can be divided into five categories; members of groups I, II, and III are widely involved in the disease resistance of plants [3-5], and members of groups IV and $\mathrm{V}$ play a role in floral organ development [6-8]. To date, the TGA transcription factor has been isolated and identified in many plants, including papaya, rice, soybean, and strawberry [9-12]. It has been confirmed that the TGA transcription factor is involved in the process of drought resistance, salt tolerance, and detoxification, and promotes root nitrate absorption [13-17].

Most TGAs in the D subfamily have the ability to improve plant resistance to pathogens and xenobiotic stress. The class II TGA2, TGA5, and TGA6 perform as key transcriptional regulators in Systemic Acquired Resistance (SAR) [18]. As salicylic acid (SA) concentration in the cell increases under pathogen infection, the NPR1 complex is decomposed and translocates into the nucleus, interacts with the TGA factors to induce the transcription of the antimicrobial $P R$ genes, and enhances general resistance [19]. (At)NPR1 transgenic rice overexpressing an $r T G A 2.1$ mutant that could no longer bind DNA or endogenous rTGA2.1 was silenced. Both types of transgenic rice displayed increased tolerance to Xanthomonas oryzae pv. Oryzae (Xoo) and had changed expression levels of $P R$ genes [20]. In addition, W-box is the cis-element of the WRKY PR1 gene promoter. Tobacco TGA2.2 can interact with the $N t W R K Y 12$ transcription factor to regulate SA-inducible $P R-1$ gene expression [21]. In the coordinated defense response of jasmonic acid (JA) and ethylene (ETH) hormones, PLANT DEFFENSIN 1.2, (PDF1.2) was used as the disease resistance marker gene. Interestingly, JA/ETH induces the impaired expression of PDF1.2 and b-CHI in the tga2/5/6 triple mutant and decreases necrotizing Botrytis cinerea sensitivity [22]. Octadecanoid-responsive arabidopsis (AP2/ERF59, ORA59) is a transactivator that works upstream of PDF1.2. Additionally, it is strongly inhibited by SA in wild-type plants, which reduces the concentration of the PDF1.2 gene [4]. It shows that TGA regulates plant disease resistance by mediating ORA59. Studies have shown that TGA transcription factors are regulated by the cytokinin (CTK)-activated transcription factor activated transcription factor 2 (ARR2) to enhance resistance to Pseudomonas syringae Pst DC3000 [23].

Fusarium wilt, also known as Panama disease, is one of the most serious banana diseases destroying banana plants and is caused by the soil-borne fungus Fusarium oxysporum f. sp. cubense (Foc). When banana plants are infected by Foc, MaNPR1A and MaNPR1B are up-regulated, and the disease-resistant variety 'GCTCV-218' has a higher and earlier expression of MaNPR1A and PR1 genes after inoculation, thereby reducing the sensitivity to Fusarium wilt [24]. Studies have shown that multiple genes involved in SA biosynthesis and downstream signal transduction pathways are suppressed in susceptible varieties and are activated in response to disease-resistant varieties after being inoculated with Foc tropical race 4 (Foc TR4) [25,26]. Since susceptible varieties inhibit the expression of genes related to SA biosynthesis and SA content, the induced resistance to Foc TR4 may be SAdependent systemic acquired resistance [27-29]. To date, the molecular mechanism of the TGA transcription factors regulating banana disease resistance through the SA signaling pathway is not yet clear.

Therefore, we hypothesize that the banana TGA transcription factor interacts with NPR1 in the SA signaling pathway to increase resistance to Foc TR4. In our study, we cloned and obtained MaTGA 8 and performed sequence analysis. The response of MaTGA8 and MaNPR1 to exogenous SA was further analyzed, and potential NPR1 interacting proteins were screened and verified through yeast plug hybridization. We also investigated the molecular functions of the overexpression of MaTGA8 in response to Foc TR4 in Arabidopsis plants. 


\section{Results}

\subsection{Analysis of MaTGA8 Gene}

A phylogenetic tree was constructed for MaTGA8 and the TGAs family of Oryza sativa L. and Arabidopsis thaliana. By comparing the transcription factors MaTGA8, Arabidopsis AtTGA, and Oryza sativa L. OsbZIP to the transcription factor family, the MaTGA8 and OsbZIP transcription factors were found to have the closest evolutionary relationship (Figure 1); among them, banana and rice were both monophytes.

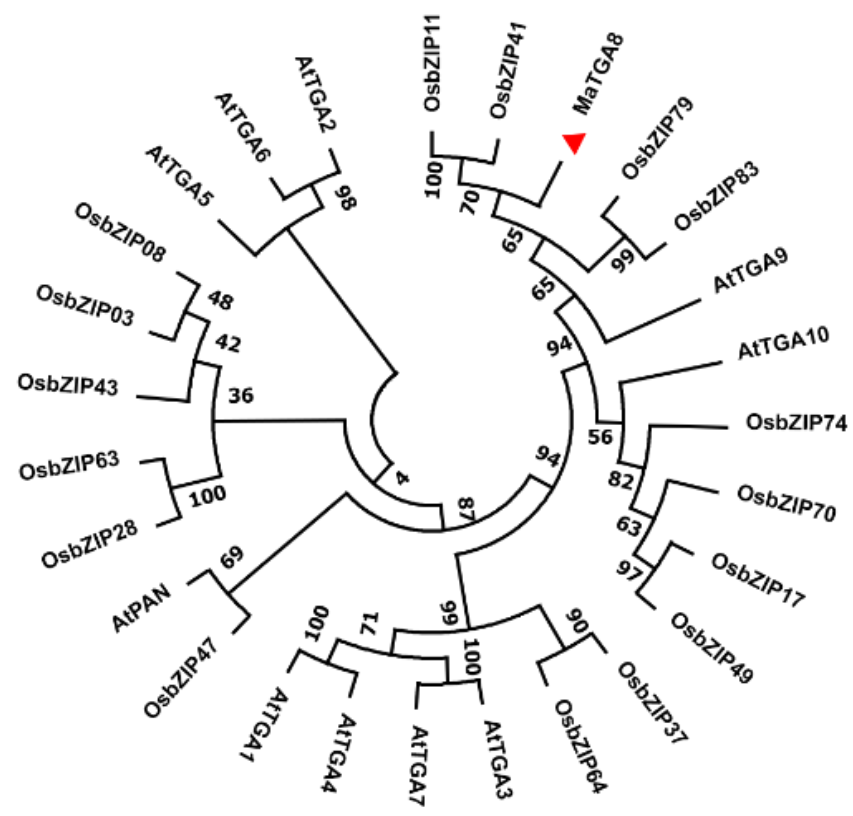

Figure 1. Phylogenetic analysis of MaTGA8 and the TGAs family of Aarabidopsis and Oryza sativa.

The protein structure of MaTGA8 was analyzed. ORF of MaTGA8 contained 504 amino acids. Further analysis through protein multiple sequence alignment indicated that the MaTGA8 contained one bZIP domain and one DOG1 domain; part of the bZIP domain was composed of 14 amino acid residues as a conserved DNA binding site, and the other part was the leucine zipper region involved in the formation of dimers (Figure 2). This indicates that MaTGA8 belongs to the bZIP transcription factor family Group D subfamily.

\subsection{Promoter Analysis for Detection of Putative Cis-Elements}

The prediction of the cis-elements found that growth and development of related cis-elements identified were MRE, the TCCC-motif, the TCT-motif, the GATA-motif, the CAT-box, the G-box, and the RY-element. MBS is a cis-element involved in drought stress. The presence of hormone related cis-elements such as the TCA-element, TGACGmotif, CGTCA-motif, GARE-motif, P-box, and ABRE in promoter region indicates the responsiveness of MaTGA8 towards SA responsiveness, MeJA responsiveness, gibberellin responsiveness, and abscisic acid reactivity (Table 1).

\subsection{Expression Level of MaTGA8 and MaNPR1 under Stress Treatments}

The Expression level of the banana MaTGA8 transcription factor gene in response to Foc TR4 was investigated by quantitative real-time PCR for susceptible varieties such as Williams as well as in disease-resistant varieties such as Nan Tianhuang. The results showed that the expression level of MaTGA8 in susceptible and disease-resistant banana variety roots was rapidly induced and significantly down-regulated under Foc TR4 infection. The expression was severely inhibited compared to inhibition at day 0 (before inoculation) (Figure 3A). 

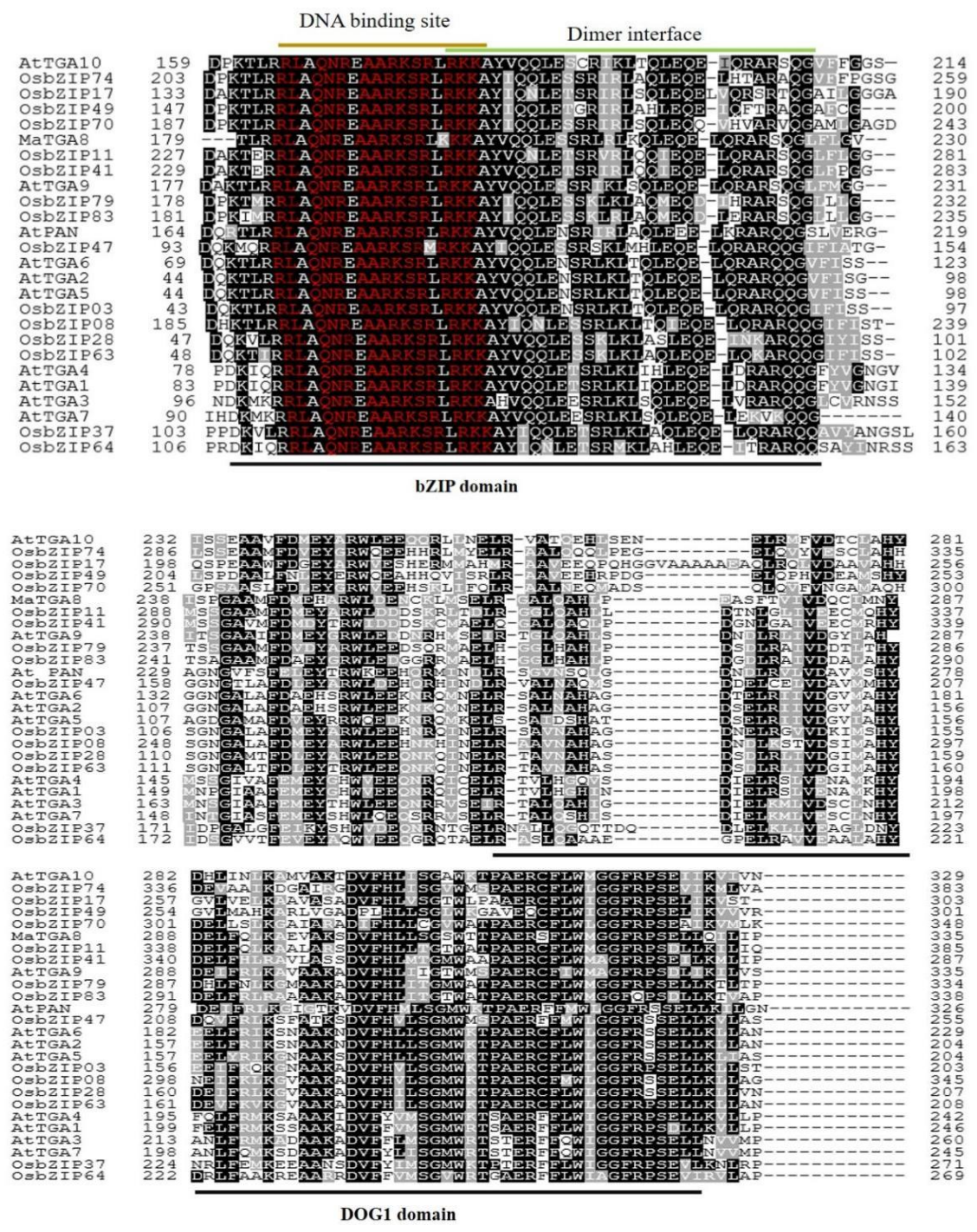

Figure 2. The protein sequence alignment of MaTGA8 and the TGAs family of Aarabidopsis and Oryza sativa. The sequences marked with thick black line are the conserved bZIP domain and the DGO1 domain. The sequences with a black background indicate completely identical residues, and the sequences with a gray background indicate similar residues.

Table 1. Prediction of the cis-acting elements of the MaTGA8 gene.

\begin{tabular}{cc}
\hline Motif & Function \\
\hline MRE & Light-response element \\
TCCC-motif & Light-response element \\
TCT-motif & Light-response element \\
G-box & Light-response element \\
GATA-motif & Light-response element \\
GT1-motif & Light-response element \\
CAT-box & Meristem expression \\
TCA-element & Involved in SA responsiveness \\
TGACG-motif & MeJA response element \\
CGTCA-motif & MeJA response elements \\
GARE-motif & Gibberellin response element \\
P-box & Gibberellin response element \\
ABRE & Abscisic acid reactive cis-acting element \\
MBS & MYB binding site involved in drought-inducibility \\
RY-element & Seed-specific regulation \\
\hline
\end{tabular}


A

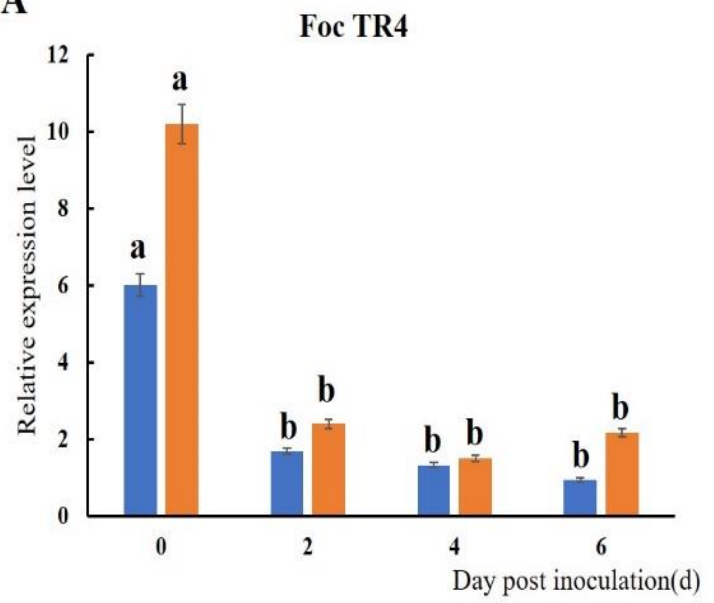

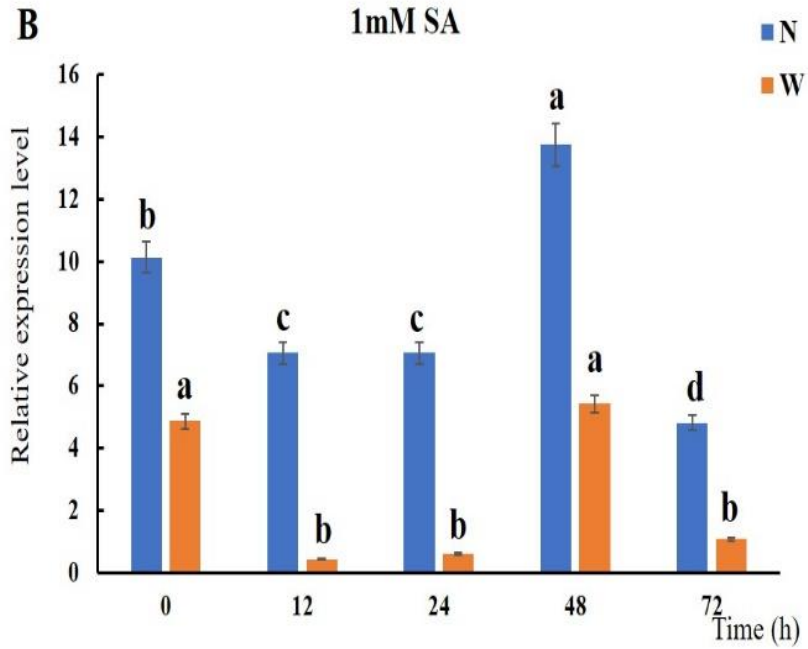

Figure 3. The expression level of MaTGA8 under Foc TR4 (A) and SA (B) treatment. N: Nan Tianhuang, W: Williams. Symbols a, b, c, etc., indicate a significant difference in the $p$ values of 0.05 .

To identity potential NPR1 interaction genes, we analyzed the response of the MaTGA8 and MaNPR1 genes to SA. MaTGA8 was significantly up-regulated at $48 \mathrm{~h}$ in the Nan Tianhuang variety, but the overall trend in both the Nan Tianhuang and Williams varieties was induced to down-regulate expression (Figure 3B), while there were obvious differences in the expression patterns of the MaNPR1 family members in both the Nan Tianhuang and Williams varieties. MaNPR1 was quickly induced by the treatment of $1 \mathrm{mMSA}$ and increased up to the highest level of 7.62-fold and 26.07-fold at 24 and $48 \mathrm{~h}$, respectively, in roots of both varieties (Figure S1). MaNPR2, MaNPR8, and MaNPR15 were significantly down-regulated in the Nan Tianhuang roots. The expression level of MaNPR2 and MaNPR8 increased up to the highest level at $48 \mathrm{~h}$ compared to the control. MaNPR15 reached its highest level at $24 \mathrm{~h}$ (Figure S1). The expression level of MaNPR3 and MaNPR11 showed an overall upward trend, increasing to the highest level of 6.42-fold and 13-fold at 48 in Williams roots and to the highest level of 10.01-fold and 7.39-fold at 12 and $24 \mathrm{~h}$ in Nan Tianhuang roots, respectively (Figure S1). The expression level of MaNPR4 increased to the highest level of 200.85 over $12 \mathrm{~h}$ in Nan Tianhuang roots (in comparison to that of the control), then declined until the end of the experiment. Similar to MaNPR4, MaNPR7 had the same expression pattern in the Nan Tianhuang roots. The expression level of MaNPR5 and MaNPR14 both showed higher induced expression at $24 \mathrm{~h}$ in the Nan Tianhuang roots (Figure S1). MaNPR9 and MaNPR13 showed higher induced expression at $24 \mathrm{~h}$ in the Williams roots, while MaNPR10 and MaNPR12 increased to the highest expression level at $12 \mathrm{~h}$ in the Nan Tianhuang roots (Figure S1).

\subsection{Yeast Two-Hybrid Assays}

The full-length coding sequence of MaTGA8 was ligated to the binding domain (BD) of GAL4 in the pGBKT7 vector and was co-expressed with the coding sequences of MaNPR3MaNPR4 and MaNPR11 fused to the GAL4 transcription activation domain (AD) of pGADT7 vector in yeast containing the AbA and x-gal reporter genes. The results show that the co-expression of MaTGA8 and MaNPR3 appeared as white colonies, while the co-expression of MaNPR4 and MaNPR11 appeared as blue colonies, and the co-expression of MaNPR4 appeared as a lighter blue (Figure 4). This shows that MaTGA8 had a weak interaction with MaNPR4 and a strong interaction with MaNPR11. These results imply that MaTGA8 could provide the DNA binding ability required by MaNPR1 to activate the MaPR gene. 

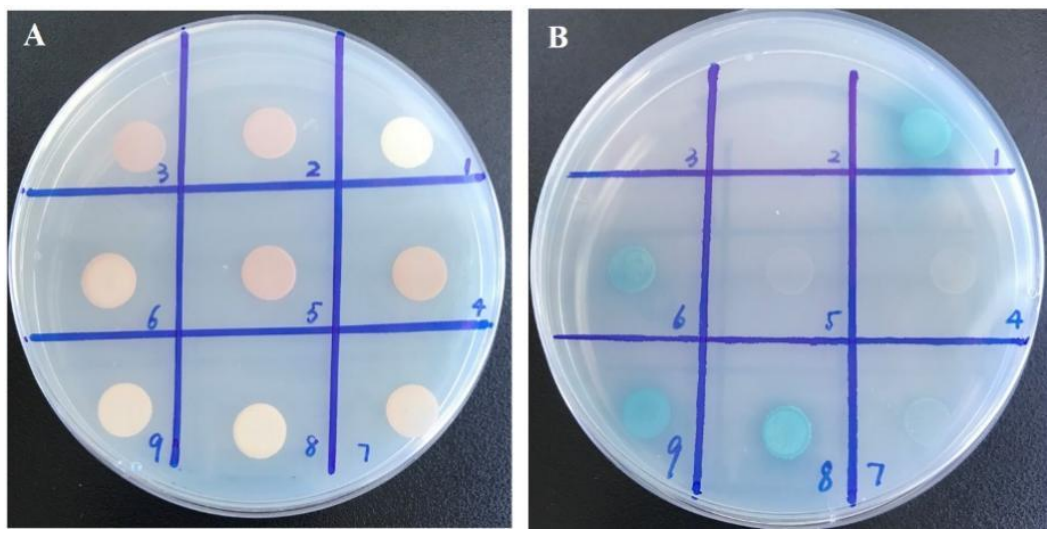

Figure 4. MaTGA8 interacts with MaNPR3, MaNPR4, and MaNPR11. Control (A); mutual verification (B); 3, 4, 5, and 6: auto-activation of pGADT7-NPR3, pGADT7-NPR4, pGADT7-NPR11, and pGBKT7-TGA8 were detected. 7, 8, 9: pGADT7-NPR3, pGADT7-NPR4, and pGADT7-NPR11 interaction with pGBKT7-TGA8 were detected. Growth of transformed yeast was evaluated on SD/-Ade/-His/-Leu/-Trp/X-a-gal/Aba medium.

\subsection{Sub-Cellular Localization of MusaTGA8}

To determine the subcellular location of MaTGA8, the full-length coding sequence was amplified via PCR and was then fused to GFP driven by the cauliflower mosaic virus (CaMV) 35S promoter. The 35S:MaTGA8-GFP vectors were then transformed into onion epidermal cells via A. tumefaciens strain GV3101, and the Laser scanning confocal microscope was used to image this phenomenon. As shown in Figure 5, the empty vector 35S: GFP was transiently expressed throughout the nucleus and cytoplasm, while the 35S: MaTGA8-GFP fusion proteins were only located in the nucleus.
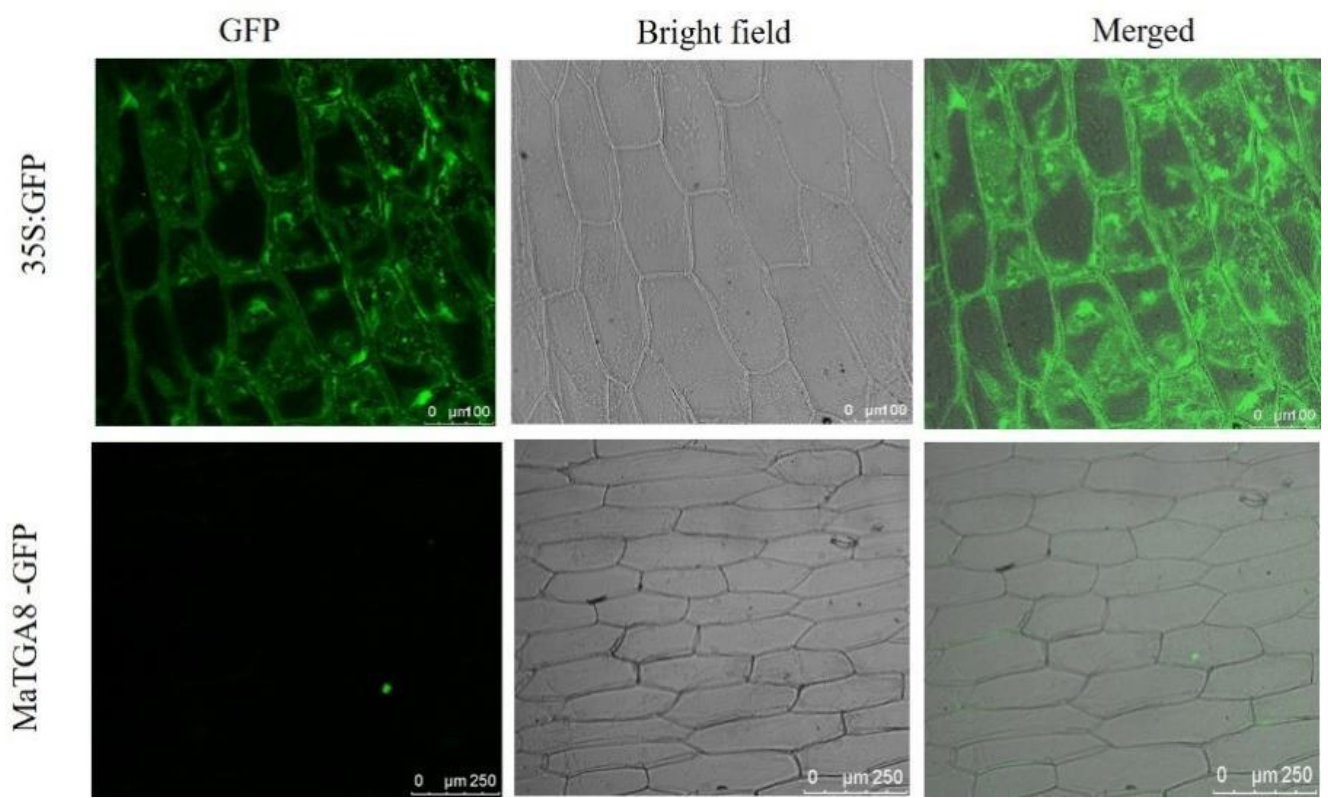

Figure 5. Subcellular localization of MaTGA8 gene.

\subsection{Overexpression of MaTGA8 Increase Foc TR4 Resistance in Arabidopsis}

To determine the expression level of MaTGA8 gene in transgenic lines, the transgenic plants were analyzed using RT-qPCR. We detected six MaTGA8-overexpression lines (tga8 OE-1 6), and the expression level of MaTGA8 was up-regulated in transgenic Arabidopsis, compared to wild-type (WT) (Figure 6A). Among them, the expression level of the MaTGA8 gene in OE-4 was 7.89 times that of the WT, and OE-3, with the lowest expression level, was 
2.95 times that of the WT (Figure 6A). This shows that the MaTGA8 gene was successfully transformed into Arabidopsis and that the mRNA was transcribed.
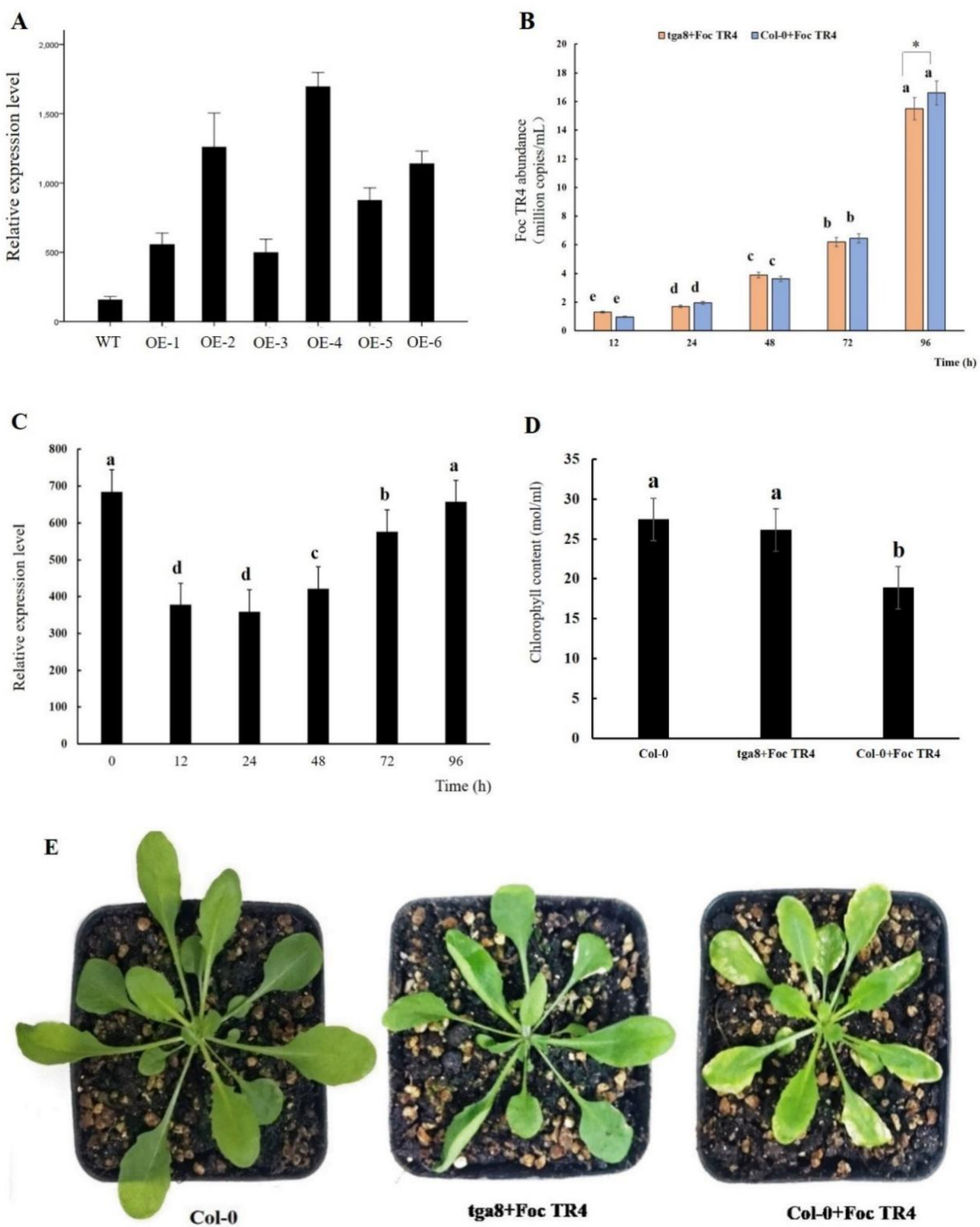

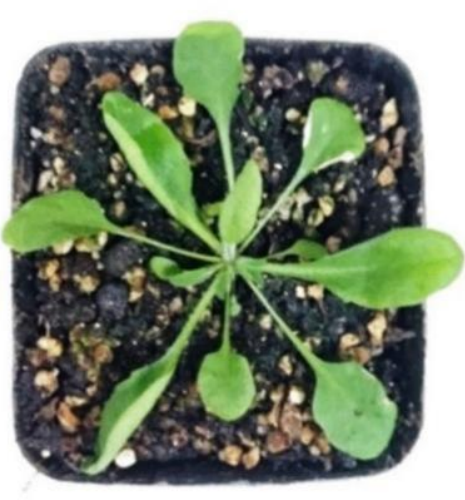

tga8+Foc TR4

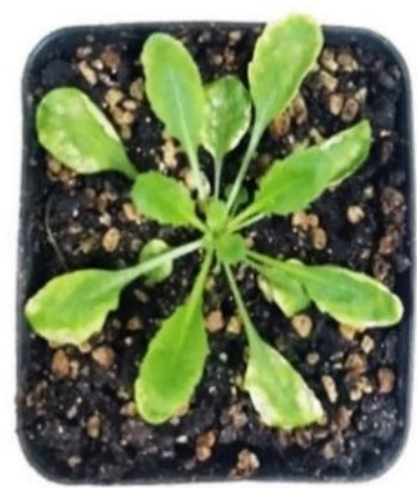

Col-10+Foc TR4

Figure 6. Improved Foc TR4 resistance in the transgenic Arabidopsis plants with the heterologous overexpression of MaTGA8. The relative expression of transgenic MaTGA8 Arabidopsis in six randomly chosen lines (A). Foc TR4 abundance in Arabidopsis plants (B). Expression level of MaTGA8 in transgenic Arabidopsis after FocTR4 infection (C). Arabidopsis chlorophyll content (D). Phenotypes of transgenic Arabidopsis and wild-type Arabidopsis after Foc TR4 infection (E). Symbols a, b, c, etc., indicate a significant difference in the $p$ values of 0.05 . 
To test whether the reduced disease symptoms in MaTGA8 transgenic plants was correlated with Foc TR4 multiplication, we checked for pathogen growth after Foc TR4 inoculation. For further analysis, the Foc TR4 qPCR detection system constructed by our laboratory was used to detect the abundance of Foc TR4 in Arabidopsis. The abundance of Foc TR4 in tga8 and WT showed an upward trend and reached $1.55 \times 10^{6}$ (copies $/ \mathrm{mL}$ ) and $1.66 \times 10^{6}$ (copies $/ \mathrm{mL}$ ) at $92 \mathrm{~h}$, respectively. This showed that Foc TR4 successfully infected Arabidopsis (Figure 6B). With the passage of transgenic Arabidopsis infection time, the mRNA level of MaTGA8 was decreased, while the overall expression level showed an upward trend (Figure 6C). Therefore, we speculated that the plant did not respond early when it was stressed by Foc TR4 and quickly generated a defensive response after recognition.

After three weeks, the Col-0 + Foc TR4 group and the tga 8 + Foc TR4 group Arabidopsis plants were smaller than the Col-0 plants, and the plants produced different degrees of yellowing symptoms in the leaves, while the yellowing symptoms of the leaves in the Col-0 + Foc TR4 group were more serious than that of the tga8+ Foc TR4 group (Figure 6E). The chlorophyll content of the Col- $0+$ Foc TR4 group was significantly different from that of the tga 8 + Foc TR4 group and Col-0 (Figure 6D). This illustrates that the overexpression the MaTGA8 gene in Arabidopsis can enhance disease resistance to Foc TR4.

\section{Discussion}

The use of resistant varieties is the most effective means to manage this disease. The resistance of Cavendish to Fusarium wilt in banana Race 1 (R1) has had an enormous impact on the banana industry, contributing to near complete Cavendish dominance in export trade, as reported by Dita et al. [30]. To date, there are no commercial cultivars that are resistant to Fusarium wilt in banana Tropical Race 4 (TR4) with similar levels of resistance from Cavendish to Fusarium wilt in banana R1; hence, the importance and benefit of considering the presence of Fusarium wilt in banana and favorable environmental conditions such as soil, climate, management agronomic, among others [31].

In the present study, a TGA transcription factor, MaTGA8, from banana was identified, and its role in the regulation of Foc TR4 tolerance in Arabidopsis plants was analyzed. MaTGA8 belongs to group-D of the bZIP transcription factor known to contain the bZIPmotif and the DOG1-motif. The plant bZIP transcription factor falls into 13 categories, and different subfamilies are involved in regulating different metabolic pathways, while transcription factors located in group $\mathrm{D}$ can participate in plant disease defense and physiological growth [32]. There is a scarcity of reports dealing with the functional analysis of the banana TGA transcription factor and their roles in regulation of defense responses. However, based on the available information, $121 \mathrm{bZIP}$ transcription factor genes were identified in the banana genome and were classified into 11 subfamilies [33]. In the phylogenetic tree, MaTGA8 has a close genetic relationship with rice OsbZIP. OsbZIP79 was reported as a negative regulator of diterpenoid phytoalexin production in rice cells [34]. Studies have shown that the cis-elements in the promoter region of a gene are closely related to the biological function of the gene [35]. Our analysis of the cis-acting elements showed that the promoter region of MaTGA8 contained the pathogen-related cis-elements, such as the P-box or GARE-motif, indicating that MaTGA8 is involved in the defense of pathogens. Therefore, we hypothesized that MaTGA8 regulates plant immunity against Foc TR4.

Non-expression of pathogenesis-related gene 1 (NPR1) is a necessary positive regulator for SA-induced pathogenesis-related (PR) gene expression and plant SAR. NPR1 function is reduced from an oligomeric state to a monomeric state and is then translocated to the nucleus after the accumulation of SA induced by pathogen infection [36]. Importantly, class II TGA and several other TGAs physically interact with NPR1, which represents the main regulator of the plant pathogen response [3,18]. TGAs and NPR1 have been fully documented as two critical components to enhance SA-dependent PR gene transcription [37]. As a set of basic region/leucine zipper motif (bZIP) factors, TGA can interact with the transcription coactivator NPR1 and can provide its DNA binding ability to activate the representative SAR marker PR gene in plants [38]. Furthermore, our results of the qRT-PCR 
analysis showed that the expression level of MaNPR1 family members varied greatly in Willams and Nan Tianhuang varieties under exogenous SA (Figure S1). The application of MaNPR3, MaNPR4, and MaNPR11, the proteins of which are extremely homologous to Arabidopsis NPR1 as prey in Y2H analysis, and the MaNPR4 and MaNPR11 proteins could effectively interact with the MaTGA8 in yeast in vivo (Figure 4). This conclusion is similar to the results of other studies $[23,39,40]$. Mutations of the NPR1 gene (SINPR1) failed to increase the resistance of tomato plants to Pseudomonas syringae, while the significant activation of SAR was recorded when the NPR1 gene was overexpressed [41].

In our study, the enhanced Foc TR4 resistance by MaTGA 8 was confirmed by its heterologous overexpression in transgenic Arabidopsis plants. Specifically, wild-type Arabidopsis and transgenic Arabidopsis under Foc TR4 infection conditions showed significantly weaker growth than uninfected wild-type Arabidopsis, while wild-type Arabidopsis plants showed more leaf wilting and lower chlorophyll content than transgenic Arabidopsis plants (Figure 6). Among rice bZIP transcription factors, OsTGA2 could interact with rice NPR1homolog proteins (NHs). Overexpression of OsTGA2 increased the resistance of rice to bacterial leaf blight [10]. Studies have shown that VvTGA1 can provide the DNA binding capacity required by $V v N P R 1$ to activate the $V v P R$ gene, and the interaction between VvNPR1 and VvTGA1 may be the cause of the effective disease resistance induced by $\beta$-aminobutyric acid (BABA) [42]. The data show that the banana MaTGA8 transcription factor interacts with MaNPR4 or MaNPR11 to activate the expression of the MaPR1 gene downstream of the SA signaling pathway and promotes the disease resistance of bananas (Figure 7).

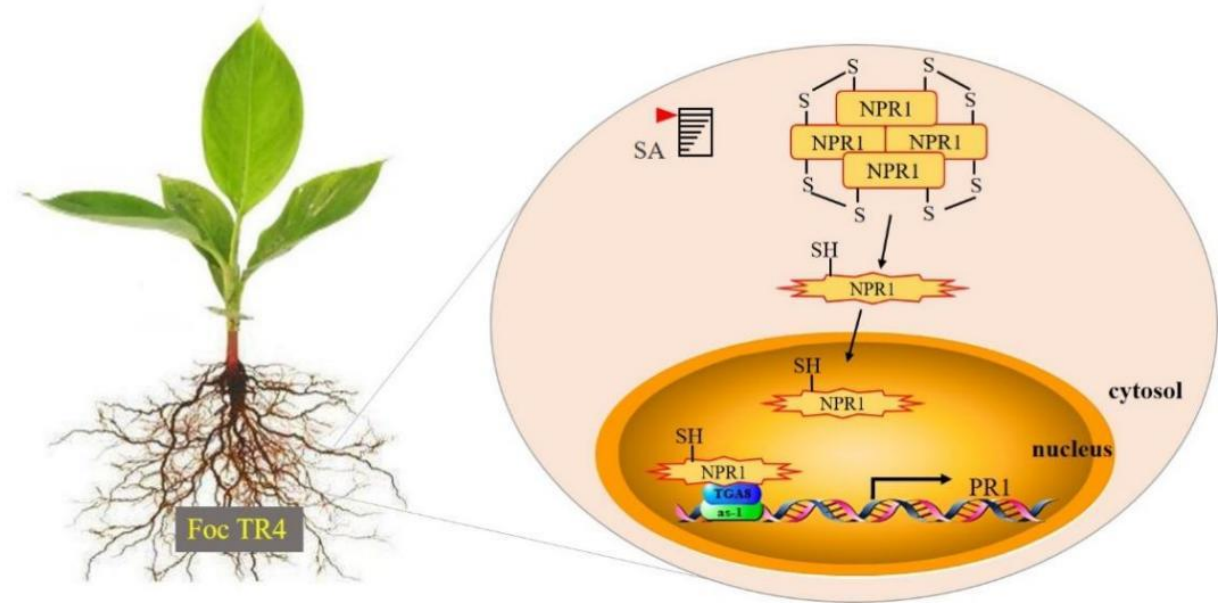

Figure 7. Working model of MaTGA8 regulates bananas to improve disease. Bananas are invaded by Foc TR4, SA accumulates in the plant, and NPR1(MaNPR4/MaNPR11) is reduced from an oligomeric state to a monomeric state and is then translocated to the nucleus, interacts with TGA factors, and promotes the expression of PR genes.

Previously, the AtTGA1-AtTGA7 of Arabidopsis plants were divided into three clades related to plant defense based on sequence homology [43]. Class II TGAs can interact with glutaredoxin GRX480 and can mediate the inhibitory effect of SA on the JA signaling pathway marker gene PDF1.2. Meanwhile, Class II TGAs can also interact with the GRAS family protein SCL14 [2,4,44]. This interaction promotes the binding of TGA to the downstream PR1 promoter, improves up-regulated expression, strengthens plant disease resistance. The SA signal pathway is administered with cytokinin, which regulates the combination of TGA3 and PR-1 promoter through ARR2 to improve plant disease resistance [23]. These studies indicate that the TGA transcription factors can mediate different signal pathways to improve plant disease resistance. However, in our study, only the SA signal pathway was discussed to regulate the process of resistance to Fusarium 
wilt in bananas. Whether other pathways regulate the process of Fusarium wilt has not been explained.

Our results could serve as the basis for future lines of research by molecular plant sciences pioneers in Colombia and Peru where Fusarium wilt in bananas (TR4) has recently been located according to Olivares et al. [45]; This would also be an essential contribution in adjacent banana areas of Latin America according to Olivares [46] in order to avoid the spread and devastation of banana plantations.

\section{Materials and Methods}

\subsection{Plant Materials and Growth Conditions}

There were two banana varieties, the Nan Tianhuang and Williams (Guangdong Shishengyuan Biological Technology Development Co., Ltd., Guangdong, China), that were used in this study. Tissue culture seedlings of the bananas were grown in nutrient soil and vermiculite mixture (3:1) in a greenhouse at $30 \pm 1{ }^{\circ} \mathrm{C}$. The young roots of banana with the first 5-leaf compound leaf unfolded were selected as the experimental materials and were treated with Fusarium oxysporum f. sp. cubense race 4 (Foc TR4) and salicylic acid $(1 \mathrm{mM})$.

The wild-type (WT) Arabidopsis Col-0 seeds were sprinkled on a nutrient substrate (soil: vermiculite 2:1 $\mathrm{v} / \mathrm{v}$ ). Arabidopsis seedlings were transplanted to new nursery pots one week after sowing and continued to cultivate in the greenhouse $(12 \mathrm{~h} \mathrm{light/dark}$ cycles and Natural light at $24^{\circ} \mathrm{C}$ ); nutrient solution was supplied once a week.

\subsection{Bacterial Strains}

The Foc TR4 was inoculated on a potato dextrose agar (PDA, Solarbio, Beijing, China) medium for 5-7 d at $28{ }^{\circ} \mathrm{C}$ and was then transferred to liquid PDA medium for 3-5 d at $28^{\circ} \mathrm{C}$ and $200 \mathrm{rpm}$ shaking culture. The Foc TR4 solution was filtered by sterilized six-layer gauze to remove the mycelium, and the spore solution concentration was adjusted to $5 \times 10^{7}$ spores $/ \mathrm{mL}$.

\subsection{The Total RNA Extraction and Quantitative Real-Time PCR ( $q$ RT-PCR)}

The plant RNA isolation was performed using a polysaccharide polyphenol plant RNA extraction kit (DP441, TIANGEN, Beijing, China). The total RNA integrity was confirmed using $1 \%$ agarose gel electrophoresis. RNA concentration and quantity were measured with the microspectrophotometer ND2000C (ThermoFisher, Waltham, MA, USA). Then, the total RNA was used for cDNA synthesis using a PrimeScript ${ }^{\mathrm{TM}} 1$ st Strand cDNA Synthesis Kit following the manufacturer's protocol (TaKaRa, Japan). For the qRT-PCR, each microcentrifuge tube contained $2 \mu \mathrm{L}$ of diluted cDNA, $10 \mu \mathrm{L}$ of TB Green Premix (TaKaRa, Japan), $1 \mu \mathrm{L}$ of gene-specific primers $(10 \mathrm{mM})$, and $6 \mu \mathrm{L} \mathrm{ddH}_{2} \mathrm{O}$. The PCR amplification procedure was as follows: predenaturation at $95{ }^{\circ} \mathrm{C}$ for $30 \mathrm{~s}$ followed by 40 cycles of $95{ }^{\circ} \mathrm{C}$ for $5 \mathrm{~s}, 55^{\circ} \mathrm{C}$ for $30 \mathrm{~s}$, and $72{ }^{\circ} \mathrm{C}$ for $30 \mathrm{~s}$. Finally, the dissociation melting curve program was set up. The specificity of the PCR products was confirmed by dissociation melting curve analysis. The housekeeping genes MaActin and AtActin were used as internal references for banana and Arabidopsis, respectively. Each sample test was set to produce three biological replications. The values of the transcript abundance were calculated using the $2^{-\triangle \Delta \mathrm{Ct}}$ method. All of the primer sequences used in this study are listed in Supplementary Table S1.

\subsection{Cloning and Sequence Analysis of MaTGA8}

The MaTGA8 gene (ID: Ma11_g13030) was amplified from banana cDNA. The cloning specific primer is described in Supplementary Table S2. The PCR amplification system was as follows: $1 \mu \mathrm{L}$ each of forward primer and reverse primer $(10 \mathrm{mM}), 2 \mu \mathrm{L}$ of diluted cDNA, $2.5 \mu \mathrm{L}$ of $10 \times$ buffer, $2 \mu \mathrm{L}$ of dNTP Mix ( $2.5 \mathrm{mmol} / \mathrm{L}), 0.25$ of LA polymerase (TaRaKa, Japan), and $\mathrm{ddH}_{2} \mathrm{O}$ were mixed to a volume of $25 \mu \mathrm{L}$. The PCR running program was $94{ }^{\circ} \mathrm{C}$ for $3 \mathrm{~min}$ followed by 35 cycles of $94{ }^{\circ} \mathrm{C}$ for $30 \mathrm{~s}, 56{ }^{\circ} \mathrm{C}$ for $30 \mathrm{~s}, 72{ }^{\circ} \mathrm{C}$ for $5 \mathrm{~min}$, and a 
final extension of $72{ }^{\circ} \mathrm{C}$ for $10 \mathrm{~min}$. The coding sequences of MaTGA8 were cloned into the pMD-18-T vector (TaRaKa, Japan) and were then transformed into E. coli DH5 $\alpha$ (Weidi Biotechnology Co., Ltd., Shanghai, China) for verification.

The sequences of MaTGA8 were used to construct a phylogenetic tree using MEGA 7.0 software (Mega Limited, Auckland, New Zealand) with the neighbor-joining method (with boot strap replications of 1000). The MaTGA8, rice, and Arabidopsis TGA proteins were aligned using multi-sequence alignment by means of ClustX (http: / / www.clustal.org / clustal2/\#Webservers accessed on 5 April 2020). To understand the role of the MaTGA8 gene in the growth and development of banana plants, we predicted the cis-acting elements of MaTGA8 using the PlantCare database (http:/ / bioinformatics.psb.ugent.be/webtools / plantcare/html/ accessed on 2 May 2020).

\subsection{Plastid Construction and Generation of Transgenic Arabidopsis Plants}

According to the restriction endonuclease sites on the expression maps of the pMD18-T and pCAMBIA-1302 vectors, two restriction endonucleases, Pst I and Kpn I (TaRaKa, Japan), were individually used to double digeste the pMD-MaTGA8 and pCAMBIA-1302 vectors. Additionally, then the full-length coding sequence of MaTGA 8 was inserted between the Pst I and Kpn I sites of the pCAMBIA-1302 vector.

To obtain stable transgenic Arabidopsis lines, the recombinant expression vector pCAMBIA-1302-MaTGA8 was transformed into the Agrobacterium tumefaciens strain GV3101 (Weidi Biotechnology Co., Ltd., Shanghai, China) and was then transformed into Arabidopsis ecotype Col-0 by means of the floral dip method [47]. The resultant T0 seedlings were screened on resistant media that contained the hygromycin and were confirmed via qRT-PCR. T3 homozygous transgenic lines were used for experiments.

\subsection{Subcellular Localization of MaTGA8}

The overexpression of the pCAMBIA-1302 vector containing 35S: MaTGA8-GFP was used to conduct the subcellular localization experiments. This expression vector was transformed into the Agrobacterium tumefaciens GV3101 strain. Square (1 cm) onion epidermal cells were cut from sterilized fresh onion bulbs and were immediately submerged into a suspension of $A$. tumefaciens at OD600 $=1$. After inoculation for $20 \mathrm{~min}$, infected onion epidermal cells were used with filter papers to absorb excess agrobacterium solution and were coincubated on 1/2 MS medium for 3 days at $25^{\circ} \mathrm{C}$. A 35S: GFP blank vector was used as a positive control. GFP imaging was performed under a laser confocal microscope (LEICA TCS SP8).

\subsection{Yeast Two-Hybrid Assays}

Two-hybrid ( $\mathrm{Y} 2 \mathrm{H})$ screening was performed according to the manufacturer's protocols using the Matchmaker ${ }^{\mathrm{TM}}$ Gold Yeast Two-Hybrid System (Clontech, TaRaKa, Japan). The full-length sequence of MaTGA8 was cloned into the bait vector pGBKT7 and was then transformed into the yeast strain Y2HGold. X-a-Gal and aureobasidin A (AbA) were used as a yeast selection marker and were added to the selection medium to reduce the autonomous activation of pGBKT7-MaTGA8. To confirm MaTGA8 and the three MaNPR1 interactions, the full-length MaNPR3, MaNPR4, and MaNPR11 was cloned into the prey vector pGADT7. Additionally, the pGADT7-MaNPR1 plasmid was then transferred to Y2HGold (containing pGBKT7-TGA8) Yeast competent by means of a co-transformation method; the positive cloned strains were cultured on a SD-Ade-His-Leu-Trp-X-a-gal-AbA solid plate.

\subsection{Foc TR4 Treatment in Transgenic Arabidopsis}

The transgenic Arabidopsis with the overexpression of MaTGA8 and the Col-0 control group were selected to conduct the Foc TR4 tolerance assays. Transgenic Arabidopsis (tga8) and Col-0 were irrigated with $30 \mathrm{~mL}$ Foc TR4 (the tga 8 + Foc TR4 and Col- 0 + Foc TR4 group) after the third week of transplantation. The phenotypic differences between the 
transgenic Arabidopsis and Col-0 were then observed after three weeks of treatment. The expression level of MaTGA8 in transgenic Arabidopsis and the abundance of Foc TR4 in Arabidopsis were also measured at the initial stage of Foc TR4 treatment.

\subsection{Statistical Analysis}

In this study, there were at least three biological replicates for all experiments. All data were analyzed using ANOVA and Student's $t$-test and were expressed as means \pm standard deviation (SD). The lowercase letters $(\mathrm{a}, \mathrm{b} \ldots)$ and asterisk $\left({ }^{*}\right)$ were used to indicate the differences between the two analysis methods at $p<0.05$.

\section{Conclusions}

The MaTGA8 gene was characterized as a nuclear localization transcription factor and belongs to the D subfamily of the bZIP family. The Foc TR4 infection triggered a defense response that was intently related to the SA-mediated immune regulatory pathway. MaNPR4 and MaNPR11 were confirmed to be the downstream target of MaTGA8 by direct protein-protein interaction. In addition, overexpression of MaTGA 8 significantly increased the resistance of transgenic Arabidopsis to Foc TR4 and slowed the growth and development of transgenic Arabidopsis. In summary, this study has shown that MaTGA8 can provide the DNA binding capacity required by MaNPR4 and MaNPR11 to activate the MaPR1 gene and can improve the disease resistance of bananas by regulating the SA-mediated signal pathway. This means that MaTGA8 may jointly regulate banana resistance through other plant hormone signaling pathways.

Supplementary Materials: The following are available online at https://www.mdpi.com/article/ 10.3390/ijms22179344/s1.

Author Contributions: P.L., T.D., J.L. and M.W. conceived the research; P.L., Y.L., W.C. and N.Z. designed and conducted the experiments; P.L., Y.L. and K.C. analyzed the data; P.L. wrote the manuscript; Y.C. and M.W. revised the manuscript. All authors have read and agreed to the published version of the manuscript.

Funding: This work was supported by the project Promotion Program for Young and Middle-aged Teacher in Science and Technology Research of Huaqiao University (ZQN-YX507), Science and technology plan project of Guangzhou (201903010010), and the Quanzhou Municipal Science and Technology Project (2019N029).

Institutional Review Board Statement: Not applicable.

Informed Consent Statement: Not applicable.

Data Availability Statement: Not applicable.

Conflicts of Interest: The authors declare that they have no conflict of interest.

\section{References}

1. Butterbrodt, T.; Thurow, C.; Gatz, C. Chromatin immunoprecipitation analysis of the tobacco PR-1a-and the truncated CaMV $35 \mathrm{~S}$ promoter reveals differences in salicylic acid-dependent TGA factor binding and histone acetylation. Plant Mol. Biol. 2006, 61, 665-674. [CrossRef] [PubMed]

2. Gatz, C. From Pioneers to Team Players: TGA Transcription Factors Provide a Molecular Link between Different Stress Pathways. Mol. Plant Microbe Interact. 2013, 26, 151-159. [CrossRef] [PubMed]

3. Zhang, Y.; Tessaro, M.J.; Lassner, M.; Li, X. Knockout Analysis of Arabidopsis Transcription Factors TGA2, TGA5, and TGA6 Reveals Their Redundant and Essential Roles in Systemic Acquired Resistance. Plant Cell 2003, 15, 2647-2653. [CrossRef] [PubMed]

4. Zander, M.; La Camera, S.; LaMotte, O.; Métraux, J.-P.; Gatz, C. Arabidopsis thaliana class-II TGA transcription factors are essential activators of jasmonic acid/ethylene-induced defense responses. Plant J. 2009, 61, 200-210. [CrossRef] [PubMed]

5. Sun, T.; Busta, L.; Zhang, Q.; Ding, P.; Jetter, R.; Zhang, Y. TGACG-BINDING FACTOR 1 (TGA1) and TGA4 regulate salicylic acid and pipecolic acid biosynthesis by modulating the expression of SYSTEMIC ACQUIRED RESISTANCE DEFICIENT 1 (SARD1) and CALMODULIN-BINDING PROTEIN 60g (CBP60g). New Phytol. 2018, 217, 344-354. [CrossRef] [PubMed]

6. Hepworth, S.; Zhang, Y.; McKim, S.; Li, X.; Haughn, G.W. BLADE-ON-PETIOLE-Dependent Signaling Controls Leaf and Floral Patterning in Arabidopsis. Plant Cell 2005, 17, 1434-1448. [CrossRef] 
7. Xu, M.; Hu, T.; McKim, S.M.; Murmu, J.; Haughn, G.W.; Hepworth, S.R. Arabidopsis BLADE-ON-PETIOLE1 and 2 promote floral meristem fate and determinacy in a previously undefined pathway targeting APETALA1 and AGAMOUS-LIKE24. Plant J. 2010, 63, 974-989. [CrossRef]

8. Maier, A.T.; Stehling-Sun, S.; Offenburger, S.-L.; Lohmann, J.U. The bZIP Transcription Factor PERIANTHIA: A Multifunctional Hub for Meristem Control. Front. Plant Sci. 2011, 2, 79. [CrossRef]

9. Espín, F.M.I.; Peraza-Echeverria, S.; Fuentes, G.; Santamaría, J.M. In silico cloning and characterization of the TGA (TGACG MOTIF-BINDING FACTOR) transcription factors subfamily in Carica papaya. Plant Physiol. Biochem. 2012, 54, 113-122. [CrossRef]

10. Moon, S.-J.; Park, H.J.; Kim, T.-H.; Kang, J.-W.; Lee, J.-Y.; Cho, J.-H.; Lee, J.-H.; Park, D.-S.; Byun, M.-O.; Kim, B.-G.; et al. OsTGA2 confers disease resistance to rice against leaf blight by regulating expression levels of disease related genes via interaction with NH1. PLoS ONE 2018, 13, e0206910. [CrossRef]

11. Ullah, I.; Magdy, M.; Wang, L.; Liu, M.; Li, X. Genome-wide identification and evolutionary analysis of TGA transcription factors in soybean. Sci. Rep. 2019, 9, 1-14. [CrossRef] [PubMed]

12. Feng, J.; Cheng, Y.; Zheng, C. Expression patterns of octoploid strawberry TGA genes reveal a potential role in response to Podosphaera aphanis infection. Plant Biotechnol. Rep. 2019, 14, 55-67. [CrossRef]

13. Behringer, C.; Bartsch, K.; Schaller, A. Safeners recruit multiple signalling pathways for the orchestrated induction of the cellular xenobiotic detoxification machinery in Arabidopsis. Plant Cell Environ. 2011, 34, 1970-1985. [CrossRef] [PubMed]

14. Du, X.; Du, B.; Chen, X.; Zhang, S.; Zhang, Z.; Qu, S. Overexpression of the MhTGA2 gene from crab apple (Malus hupehensis) confers increased tolerance to salt stress in transgenic apple (Malus domestica). J. Agric. Sci. 2013, 152, 634-641. [CrossRef]

15. Alvarez, J.M.; Riveras, E.; Vidal, E.A.; Gras, D.E.; Contreras-López, O.; Tamayo, K.P.; Aceituno, F.; Gómez, I.; Ruffel, S.; Lejay, L.; et al. Systems approach identifies TGA1 and TGA4 transcription factors as important regulatory components of the nitrate response of Arabidopsis thaliana roots. Plant J. 2014, 80, 1-13. [CrossRef] [PubMed]

16. Canales, J.; Contreras-López, O.; Álvarez, J.M.; Gutiérrez, R.A. Nitrate induction of root hair density is mediated by TGA1/TGA4 and CPC transcription factors in Arabidopsis thaliana. Plant J. 2017, 92, 305-316. [CrossRef]

17. Li, B.; Liu, Y.; Cui, X.Y.; Fu, J.D.; Zhou, Y.B.; Zheng, W.J.; Lan, J.H.; Jin, L.G.; Chen, M.; Ma, Y.Z. Genome-Wide characterization and expression analysis of soybean TGA transcription factors identified a novel TGA gene involved in drought and salt tolerance. Front. Plant Sci. 2019, 10, 549. [CrossRef]

18. Fu, Z.Q.; Dong, X. Systemic Acquired Resistance: Turning Local Infection into Global Defense. Annu. Rev. Plant Biol. 2013, 64, 839-863. [CrossRef]

19. Wither, J.; Dong, X. Posttranslational modifications of NPR1: A single protein playing multiple roles in plant immunity and physiology. PLoS Pathog. 2016, 12, e1005707. [CrossRef]

20. Fitzgerald, H.A.; Canlas, P.E.; Chern, M.-S.; Ronald, P.C. Alteration of TGA factor activity in rice results in enhanced tolerance to Xanthomonas oryzae pv. oryzae. Plant J. 2005, 43, 335-347. [CrossRef]

21. Van Verk, M.C.; Neeleman, L.; Bol, J.F.; Linthorst, H.J.M. Tobacco Transcription Factor NtWRKY12 Interacts with TGA2.2 in vitro and in vivo. Front. Plant Sci. 2011, 2, 32. [CrossRef]

22. Zander, M.; Thurow, C.; Gatz, C. TGA transcription factors activate the salicylic acid-suppressible branch of the ethylene-induced defense program by regulating ORA59 expression. Plant Physiol. 2014, 165, 1671-1683. [CrossRef] [PubMed]

23. Choi, J.; Huh, S.U.; Kojima, M.; Sakakibara, H.; Paek, K.-H.; Hwang, I. The Cytokinin-Activated Transcription Factor ARR2 Promotes Plant Immunity via TGA3/NPR1-Dependent Salicylic Acid Signaling in Arabidopsis. Dev. Cell 2010, 19, $284-295$. [CrossRef] [PubMed]

24. Endah, R.; Beyene, G.; Kiggundu, A.; Berg, N.V.D.; Schlüter, U.; Kunert, K.; Chikwamba, R. Elicitor and Fusarium-induced expression of NPR1-like genes in banana. Plant Physiol. Biochem. 2008, 46, 1007-1014. [CrossRef] [PubMed]

25. Wang, Z.; Jia, C.; Li, J.; Huang, S.; Xu, B.; Jin, Z. Activation of salicylic acid metabolism and signal transduction can enhance resistance to Fusarium wilt in banana (Musa acuminata L. AAA group, cv. Cavendish). Funct. Integr. Genom. 2014, 15, 47-62. [CrossRef] [PubMed]

26. Sun, J.; Zhang, J.; Fang, H.; Peng, L.; Wei, S.; Li, C.; Zheng, S.; Lu, J. Comparative transcriptome analysis reveals resistance-related genes and pathways in Musa acuminata banana 'Guijiao 9' in response to Fusarium wilt. Plant Physiol. Biochem. 2019, 141, 83-94. [CrossRef]

27. Pieterse, C.M.; van der Does, D.; Zamioudis, C.; Leon-Reyes, A.; Van Wees, S. Hormonal modulation of plant immunity. Annu. Rev. Cell Dev. Biol. 2012, 28, 489-521. [CrossRef]

28. Bai, T.-T.; Xie, W.-B.; Zhou, P.-P.; Wu, Z.-L.; Xiao, W.-C.; Zhou, L.; Sun, J.; Ruan, X.-L.; Li, H.-P. Transcriptome and Expression Profile Analysis of Highly Resistant and Susceptible Banana Roots Challenged with Fusarium oxysporum f. sp. cubense Tropical Race 4. PLoS ONE 2013, 8, e73945. [CrossRef]

29. Li, W.; Li, C.; Sun, J.; Peng, M. Metabolomic, Biochemical, and Gene Expression Analyses Reveal the Underlying Responses of Resistant and Susceptible Banana Species during Early Infection with Fusarium oxysporum f. sp. cubense. Plant Dis. 2017, 101, 534-543. [CrossRef]

30. Dita, M.; Barquero, M.; Heck, D.W.; Mizubuti, E.S.G.; Staver, C.P. Fusarium Wilt of Banana: Current Knowledge on Epidemiology and Research Needs Toward Sustainable Disease Management. Front. Plant Sci. 2018, 9, 1468. [CrossRef] [PubMed] 
31. Olivares, B.O.; Araya-Alman, M.; Acevedo-Opazo, C.; Rey, J.C.; Cañete-Salinas, P.; Kurina, F.G.; Balzarini, M.; Lobo, D.; Navas-Cortés, J.A.; Landa, B.B.; et al. Relationship between Soil Properties and Banana Productivity in the Two Main Cultivation Areas in Venezuela. J. Soil Sci. Plant Nutr. 2020, 20, 2512-2524. [CrossRef]

32. Dröge-Laser, W.; Snoek, B.L.; Snel, B.; Weiste, C. The Arabidopsis bZIP transcription factor family-An update. Curr. Opin. Plant Biol. 2018, 45, 36-49. [CrossRef]

33. Hu, W.; Wang, L.; Tie, W.; Yan, Y.; Ding, Z.; Liu, J.; Li, M.; Peng, M.; Xu, B.; Jin, Z. Genome-wide analyses of the bZIP family reveal their involvement in the development, ripening and abiotic stress response in banana. Sci. Rep. 2016, 6, 30203. [CrossRef] [PubMed]

34. Miyamoto, K.; Nishizawa, Y.; Minami, E.; Nojiri, H.; Yamane, H.; Okada, K. Overexpression of the bZIP transcription factor OsbZIP79 suppresses the production of diterpenoid phytoalexin in rice cells. J. Plant Physiol. 2014, 173, 19-27. [CrossRef]

35. Cheng, M.-C.; Liao, P.-M.; Kuo, W.-W.; Lin, T.-P. The Arabidopsis ETHYLENE RESPONSE FACTOR1 Regulates Abiotic StressResponsive Gene Expression by Binding to Different cis-Acting Elements in Response to Different Stress Signals. Plant Physiol. 2013, 162, 1566-1582. [CrossRef] [PubMed]

36. Mou, Z.; Fan, W.; Dong, X. Inducers of Plant Systemic Acquired Resistance Regulate NPR1 Function through Redox Changes. Cell 2003, 113, 935-944. [CrossRef]

37. Després, C.; Chubak, C.; Rochon, A.; Clark, R.; Bethune, T.; Desveaux, D.; Fobert, P.R. The Arabidopsis NPR1 disease resistance protein is a novel cofactor that confers redox regulation of DNA binding activity to the basic domain/leucine zipper transcription factor TGA1. Plant Cell 2003, 15, 2181-2191. [CrossRef] [PubMed]

38. Zhang, Y.; Fan, W.; Kinkema, M.; Li, X.; Dong, X. Interaction of NPR1 with basic leucine zipper protein transcription factors that bind sequences required for salicylic acid induction of the PR-1 gene. Proc. Natl. Acad. Sci. USA 1999, 96, 6523-6528. [CrossRef]

39. Lindermayr, C.; Sell, S.; Müller, B.; Leister, D.; Durner, J. Redox Regulation of the NPR1-TGA1 System of Arabidopsis thaliana by Nitric Oxide. Plant Cell 2010, 22, 2894-2907. [CrossRef] [PubMed]

40. Stotz, H.U.; Findling, S.; Nukarinen, E.; Weckwerth, W.; Mueller, M.J.; Berger, S. A tandem affinity purification tag of TGA2 for isolation of interacting proteins in Arabidopsis thaliana. Plant Signal. Behav. 2014, 9, e972794. [CrossRef]

41. Xiao, S.; Chye, M.-L. Overexpression of Arabidopsis ACBP3 Enhances NPR1-Dependent Plant Resistance to Pseudomonas syringe pv tomato DC3000. Plant Physiol. 2011, 156, 2069-2081. [CrossRef] [PubMed]

42. Wang, K.; Li, C.; Lei, C.; Jiang, Y.; Qiu, L.; Zou, X.; Zheng, Y. $\beta$-aminobutyric acid induces priming defence against Botrytis cinerea in grapefruit by reducing intercellular redox status that modifies posttranslation of VvNPR1 and its interaction with VvTGA1. Plant Physiol. Biochem. 2020, 156, 552-565. [CrossRef] [PubMed]

43. Xiang, C.; Miao, Z.; Lam, E. DNA-binding properties, genomic organization and expression pattern of TGA6, a new member of the TGA family of bZIP transcription factors in Arabidopsis thaliana. Plant Mol. Biol. 1997, 34, 403-415. [CrossRef] [PubMed]

44. Fode, B.; Siemsen, T.; Thurow, C.; Weigel, R.; Gatz, C. The Arabidopsis GRAS protein SCL14 interacts with class II TGA transcription factors and is essential for the activation of stress-inducible promoters. Plant Cell 2008, 20, 3122-3135. [CrossRef]

45. Olivares, B.; Rey, J.; Lobo, D.; Navas-Cortés, J.; Gómez, J.; Landa, B. Fusarium Wilt of Bananas: A Review of Agro-Environmental Factors in the Venezuelan Production System Affecting Its Development. Agronomy 2021, 11, 986. [CrossRef]

46. Olivares Campos, B.; Paredes, F.; Rey, J.; Lobo, D.; Galvis-Causil, S. The relationship between the normalized difference vegetation index, rainfall, and potential evapotranspiration in a banana plantation of Venezuela. Sains Tanah J. Soil Sci. Agroclimatol. 2021, 18, 58-64. [CrossRef]

47. Narusaka, M.; Shiraishi, T.; Iwabuchi, M.; Narusaka, Y. The floral inoculating protocol: A simplified Arabidopsis thaliana transformation method modified from floral dipping. Plant Biotechnol. 2010, 27, 349-351. [CrossRef] 